\title{
Linear array arrangement for microwave tomography
}

\author{
G. Peronnet and J. Ch. Bolomey \\ Groupe d'Electromagnétisme, Laboratoire des Signaux et Systèmes \\ CNRS/ESE, Gif Sur Y vette, France \\ L. Jofre \\ Escola Tecnica Superior d'Enginyers de Telecomunicacio, Barcelona, Spain
}

(Received January 3, 1984; revised March 22, 1984; accepted March 26, 1984.)

\begin{abstract}
Recent developments both in measurement techniques and reconstruction algorithms have shown the possibility of quasi-real time microwave tomography. These technical developments always require more sophisticated experimental arrangements in order to reduce the microwave circuits complexity, decrease the measurement time, and improve the resolution. This paper deals with the use of crossed linear arrays convenient for tomography by transmission. Numerical simulations and initial experimental results show the relative advantages of such arrangements as compared to the more classical two-dimensional arrays.
\end{abstract}

\section{INTRODUCTION}

Like $\mathrm{X}$ rays and ultrasonic waves, microwaves may be used for biomedical imagery and tomography [Larsen and Jacobi, 1979, 1980]. Operating frequencies of the order of a few gigahertz result from a compromise between resolution and attenuation. At such frequencies, quasi-real time computerized tomography of the human body should be achieved by using low-cost equipment.

Experiments have been conducted at $3 \mathrm{GHz}$ on isolated animal organs and have confirmed that resolutions of the order of $6 \mathrm{~mm}$ can be reached in highwater-content media [Bolomey et al., 1982; Peronnet et al., 1983]. The imaging technique described in the above-mentioned papers was a tomographic process using a two-dimensional apertures arrangement. It appears then that it would be interesting to evaluate the characteristics of a more simple arrangement consisting of a crossed linear array setup which could be used for the same imaging purpose.

Linear crossed arrays such as Mills cross or quad array have already been used for radio detection or imaging purposes and acoustic holography [Bennett et al., 1979; Milder and Wells, 1970; Slattery, 1966].

One advantage of this kind of system is the reduced number of required microwave elements. This

Copyright 1984 by the American Geophysical Union.

Paper number 4S0505.

0048-6604/84/004S-0505\$08.00 property allows us to insert them in more complicated configurations and to utilize them in conjunction with other systems. As an example, a trial application to remote temperature control during a hyperthermia process is considered and evaluated.

The first part of this paper is devoted to a short description of the principles of microwave imaging by means of two-dimensional arrays. In a similar way, a description of the principles of microwave imaging by means of crossed linear arrays is given. The two methods are then compared in terms of resolution, and results from a first application of linear arrays are shown.

\section{IMAGING BY MEANS OF TWO-DIMENSIONAL ARRAYS}

Diffraction microwave imaging can be compared to optical holography [Mueller et al., 1978; Porter and Devaney, 1982]. Amplitude/phase probing of the diffracted waves allows the reconstruction of slices of a tested object in terms of equivalent current density. The resulting image depends then on the kind of illumination.

By using an integral representation, the relation between the measured diffracted field $E$ and the equivalent current density $J$ inside the object is given by (1).

$$
\begin{gathered}
\mathbf{E}(x, y, z)=[1 /(4 \pi j \omega \varepsilon)]\left(\operatorname{grad} \operatorname{div}+k^{2}\right) \\
\cdot \|_{V} \mathbf{J}(X, Y, Z) G\left(M, M^{\prime}\right) d V
\end{gathered}
$$




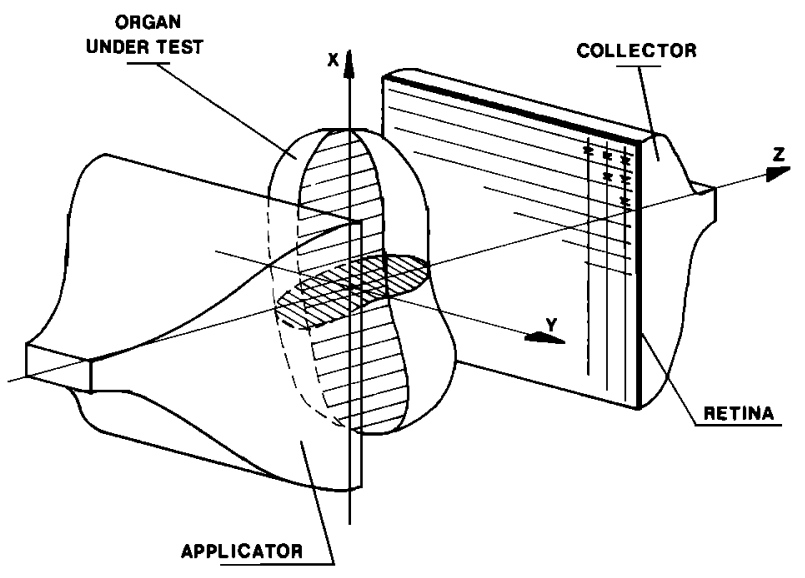

Fig. 1. General arrangement for microwave imaging by means of two-dimensional arrays.

where $G\left(M, M^{\prime}\right)$ is the three-dimensional free space Green's function of the medium surrounding the object with propagation constant $k$.

In the case of a plane wave illumination propagating in the direction normal to the two-dimensional probing surface as described in Figure 1, relation (1) can be reversed if the polarization changes are neglected and becomes the scalar relation

$J^{*}(X, Y, Z)=F_{\alpha, \beta}^{-1}\left\{S(\alpha, \beta ; Z-z) F_{x, y}[E(x, y, z)]\right\}$

where $Z-z$ is the distance between the sampling area and the plane where the current density has to be calculated, $\alpha$ and $\beta$ are the spectral coordinates associated with the spatial coordinates $x$ and $y, F_{x, y}$ is the two-dimensional direct Fourier transform operator (applied to $x$ and $y), F_{\alpha, \beta}^{-1}$ is the twodimensional inverse Fourier transform operator (applied to $\alpha$ and $\beta$ ), $E$ is the measured diffracted electric field, $J^{*}$ is the reconstructed equivalent current density, and

$$
\begin{gathered}
S(\alpha, \beta ; Z-z)=k\left(k^{2}-\alpha^{2}-\beta^{2}\right)^{1 / 2} /\left(k^{2}-\alpha^{2}\right) \\
\cdot \exp \left[j\left(k^{2}-\alpha^{2}-\beta^{2}\right)^{1 / 2}(Z-z)\right]
\end{gathered}
$$

with $j^{2}=-1$.

It has to be noticed that equivalent current density $J$ depends both on total electric field inside the test objects and on their dielectric characteristics. Due to the different approximations and to the fact that the measurement area is bounded, the reconstructed current density $J^{*}$ may be written as the convolution product of an impulse response $P_{1}$ and the exact current density $J$ :

$$
J^{*}(X, Y, Z)=P_{1}(X, Y, Z) * J(X, Y, Z)
$$

$P_{1}(X, Y, Z)$ corresponds to a spot which, spread in all directions, limits the resolution. Expressed as a function of the wavelength in the surrounding medium, typical resolutions in $X$ and $Y$ directions are $\lambda / 2$ and $2 \lambda$ in $Z$ direction.

Such an imaging process has been recently used for biomedical imaging purposes [Peronnet et al., 1983]. Field probing at $3 \mathrm{GHz}$ was achieved through a modulated scattering technique by means of a twodimensional array composed of a retina mode of $N \times N$ diodes in front of a horn (Figure 1). Diodes are successively fed through electronic scanning. The distance between two elements must be smaller or equal to half a wavelength in the surrounding medium, in this case water. At $3 \mathrm{GHz}$ this wavelength is $1.2 \mathrm{~cm}$. Array dimensions are therefore reasonable (about $20 \mathrm{~cm} \times 20 \mathrm{~cm}$ ).

Isolated organs such as kidneys of pigs and horses have been investigated. As expected, spatial transverse resolutions of $6 \mathrm{~mm}$ have been obtained. Typical acquisition rates of a few milliseconds per point have been reached.

\section{IMAGING BY MEANS OF CROSSED LINEAR ARRAYS}

In the system described here, the two crossed arrays acting as radiator and receiver are not located in the same plane. The first one is a linear array of dipoles and the second one a linear array composed of diodes in front of a collecting horn (Figure 2). As one of the dipoles is activated, field probing is achieved on the entire array which provides $N \times N$ samples.

The relation between the measured diffracted field and the reconstructed equivalent current density is different from (2). One can interpret (2) as the focalization of the diffracted wave at the point where the

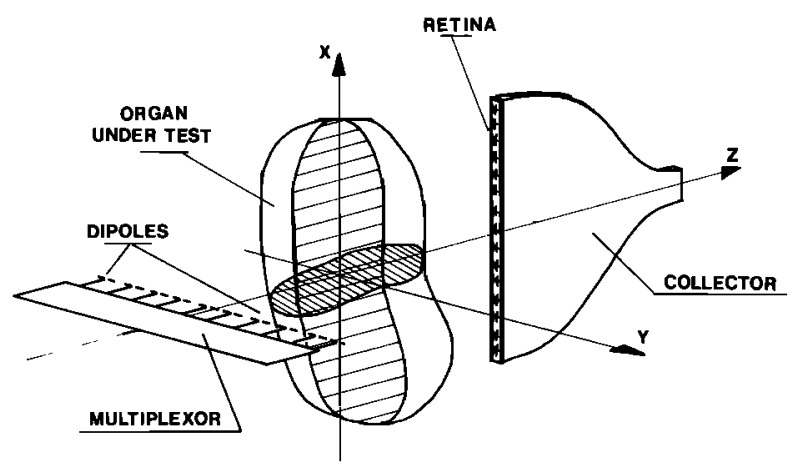

Fig. 2. General arrangement for microwave imaging by means of two crossed linear arrays. 
image is to be calculated. The focalization operator $S$ transforms the divergent plane wave measured into a convergent one propagating in the reverse direction.

The purpose of this article is to describe an operator that is equivalent to $S$ but for the linear array setup. At this state of the art it was enough for us to show that this new operator was convenient for focalization operations and had good characteristics in term of resolution as indicated later in the present paper. In addition, experimental results confirm the choices that have been made.

With a linear distribution of current, waves are no longer spherical but are cylindrical. As is well known, an infinite uniform linear current distribution creates a field that may be expressed by

$$
E(X, Y, Z)=\eta I_{0} H_{0}^{(2)}\left[k\left(X^{2}+Z^{2}\right)^{1 / 2}\right]
$$

where $H_{0}^{(2)}$ is the Hankel function of second kind with a current colinear to the $y$ axis, $\eta$ is the wave impedance in the surrounding medium, and $I_{0}$ is the amplitude of the current on the radiating linear array.

It can be shown that an operator similar to $S$ that is able to transform a divergent cylindrical wave into a convergent one is proportional to $C_{1}$ such that

$$
\begin{gathered}
C_{1}\left[\beta ;\left(X^{2}+Z^{2}\right)^{1 / 2}\right]=\frac{k}{k^{2}-\alpha^{2}} \\
\cdot H_{0}^{(1)}\left[\left(k^{2}-\beta^{2}\right)^{1 / 2}\left(X^{2}+Z^{2}\right)^{1 / 2}\right]
\end{gathered}
$$

To understand how crossed linear arrays may be used for an imaging purpose, we can first consider that the emitting array described by Figure 2 is focalized on point $M(X, Y, Z)$. In a realistic setup the emitting array is not focalized on the point to be reconstructed. Nevertheless, it can be noticed that this could be achieved by applying proper amplitude

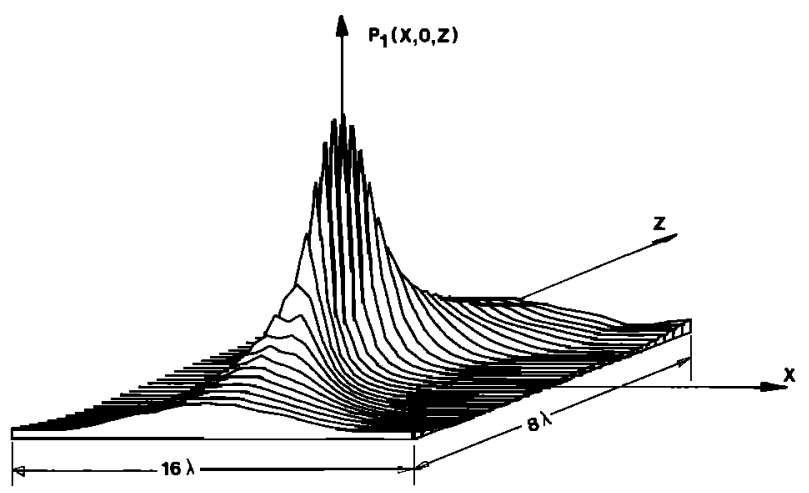

Fig. 3. Impulse response in $Y$ plane for the two-dimensional array setup.

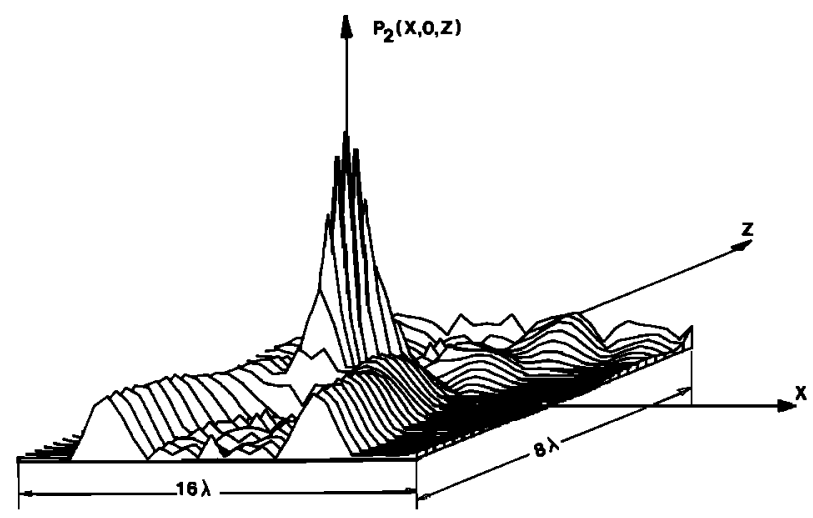

Fig. 4. Impulse response in $Y$ plane for the crossed linear array setup.

and phase weighting coefficients to the emitting elements. If the polarization changes are neglected, the weighting coefficients can be deduced from $C_{1}$.

If now we want to focalize at the same point the diffracted field measured by the receiving array located at $z$, the focalization operator is then

$$
\begin{gathered}
C_{2}\left\{\alpha ;\left[Y^{2}+(Z-z)^{2}\right]^{1 / 2}\right\}=\left[k /\left(k^{2}-\beta^{2}\right)\right] H_{0}^{(1)} \\
\cdot\left\{\left(k^{2}-\alpha^{2}\right)^{1 / 2}\left[Y^{2}+(Z-z)^{2}\right]^{1 / 2}\right\}
\end{gathered}
$$

If now all the emitting elements create the same incident wave, the total focalization operator to apply to the measured electric field $E$ is

$$
C_{3}\left(\alpha, \beta ; \rho_{1}, \rho_{2}\right)=C_{1}\left(\beta ; \rho_{1}\right) C_{2}\left(\alpha ; \rho_{2}\right)
$$

where $\rho_{1}=\left(X^{2}+Z^{2}\right)^{1 / 2}$ and $\rho_{2}=\left[Y^{2}+(Z-\right.$ $\left.z)^{2}\right]^{1 / 2}$ if $X, Y$, and $Z$ are the coordinates of the focalized point.

The generalized imaging algorithm becomes

$$
\begin{gathered}
J^{*}(X, Y, Z)=F_{a}^{-1}\left\{C _ { 2 } ( \alpha ; \rho _ { 2 } ) F _ { x } \left\{F_{\beta}^{-1}\right.\right. \\
\left.\left.\cdot\left[C_{1}\left(\beta ; \rho_{1}\right) F_{y}[E(x, y, z)]\right]\right\}\right\}
\end{gathered}
$$

where $F_{x}$ and $F_{y}$ are the one-dimensional direct Fourier transform (applied to $x$ and $y$ coordinates), $F_{a}^{-1}$ and $F_{\beta}^{-1}$ are the one-dimensional inverse Fourier transform (applied to $\alpha$ and $\beta$ coordinates), $E(x, y, z)$ is the diffracted field measured at point $x$ of the receiving antenna located at $z$ when element $y$ of the emitting antenna is active, and $J^{*}(X, Y, Z)$ is the reconstructed equivalent current density at point $M(X, Y, Z)$.

Equation (9) has to be compared with equation (2). They both are relevant to a tomographic process because they allow one to obtain images, expressed in terms of distribution of equivalent current density 


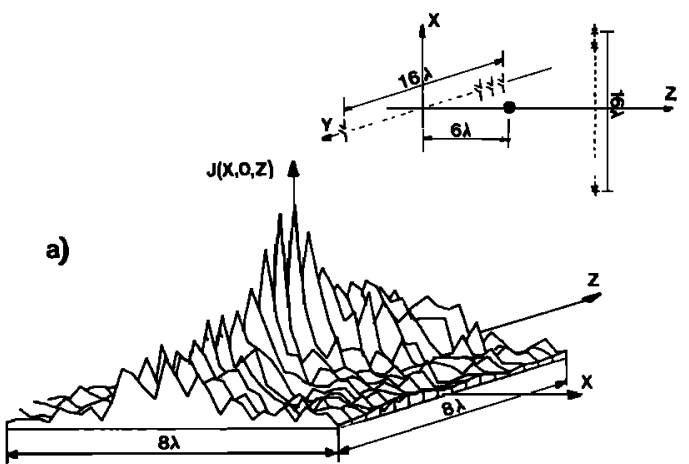

Fig. 5a. Reconstructed equivalent current density for a metallic spherical object (diameter $1.2 \mathrm{~cm}$ ) placed at $6 \lambda$ from the emitting array $\left(z_{m}=12 \lambda\right)$.

calculated for slices in any direction into the tested object.

As with the classical two-dimensional imaging process, the reconstructed current density can be expressed as the convolution product of an impulse response $P_{2}$ by the exact current density at point $M$ :

$$
\begin{gathered}
J^{*}(X, Y, Z)=P_{2}(X, Y, Z) * J(X, Y, Z) \\
\text { RESULTS }
\end{gathered}
$$

\section{RESULTS}

Figures 3 and 4 show the variations of $P_{1}$ and $P_{2}$ for the corresponding imaging setup consisting of $32 \times 32$ sampling points with a sampling interval of half a wavelength at $3 \mathrm{GHz}$. The curves of these figures are relevant to the plane $y=0$ for a simulated point object at $x=y=0$ and $Z=6 \lambda$.

Figure 5 shows initial experimental results corresponding to the same configuration for a metallic spherical object with diameter $\lambda$. It appears that in both cases, transverse resolution (in the $x$ direction) is better than longitudinal resolution (in the $z$ direction), but the latter is slightly better with crossed linear arrays. Transverse resolution is half a wavelength in the two configurations.

These initial results show that with a very reduced number of elements and a not much more complicated imaging algorithm, the linear crossed array ar-

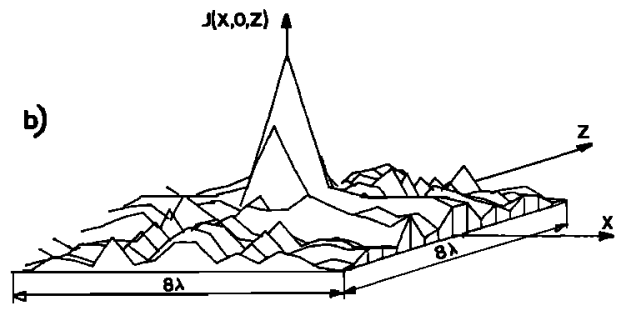

Fig. $5 b$. Same as Figure $5 a$ but for four identical views around the object.

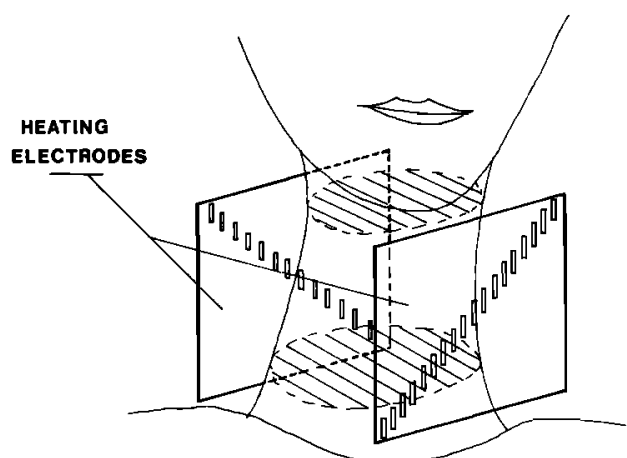

Fig. 6. Insertion of two crossed linear arrays into a hyperthermia system.

rangement gives the same result in terms of resolution as with the two-dimensional arrays. Power considerations must be taken into account in the choice of the arrangement for an imaging system because of the low sensitivity of crossed linear array systems.

Figure 6 describes a possible application relevant to the difficult problem of remote temperature sensing during a hyperthermia process. It has been shown that microwave images are sensitive to temperature changes because of the temperature dependence of tissue dielectric constants [Bolomey et al., 1983]. Resolutions of the order of $1^{\circ}$ have been observed. This particular sensitivity may be applied to noninvasive temperature sensing in the human body and used to control heating effects during a hyperthermia process.

Among the various existing radiofrequency hyperthermia systems is the one consisting of two capacitive electrodes that are applied to the patient (Figure 6). Typical heating frequencies are 13 and $27 \mathrm{MHz}$.

The suggested idea is to insert microwave slot an-

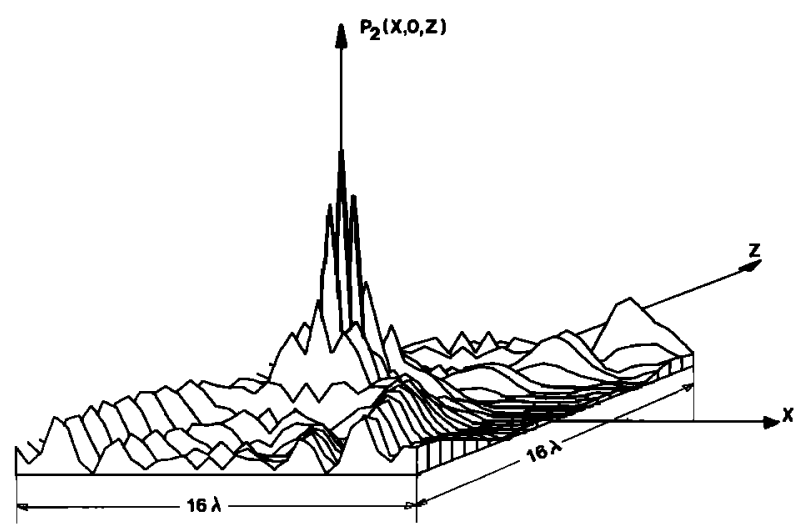

Fig. 7. Impulse response in $Y$ plane corresponding to the imaging system described by Figure 5. 
tennas in one of the capacitive electrodes that will act as the emitting linear array and to insert slots loaded by diodes in the other one that will act as the receiving linear array. By using the imaging process described by (9), it is then possible to evaluate the impulse response of such a system. The result is given in Figure 7 for the same configuration as for Figures 3 and 4 in the case of $10 \mathrm{~cm} \times 10 \mathrm{~cm}$ electrodes.

\section{CONCLUSION}

It has been shown that crossed linear arrays must be used for imaging purposes in biological media. The resolutions of such systems have been compared with the classical two-dimensional imaging system; they are quite similar in both cases. But the simplicity of the required circuit makes this kind of imaging easier than with a two-dimensional arrangement. Its application to the remote temperature sensing during a hyperthermia process has then been considered. For typical capacitive electrodes, one should expect a spatial resolution of approximately 1 $\mathrm{cm}$ and a temperature resolution of about $1^{\circ}$.

\section{REFERENCES}

Bennett, J. C., S. N. M. Al-Adhani, and A. P. Anderson, A novel radar array and its imaging properties, IEEE Trans. Antennas Propag., 27(4), 567-570, 1979.
Bolomey, J. C., A. Izadnegahdar, L. Jofre, G. Peronnet, C. Pichot, and $M$. Solaimani, Microwave diffraction tomography for biomedical applications, IEEE Trans. Microwave Theory Tech., 30(11), 1998-2000, 1982.

Bolomey, J. C., L. Jofre, and G. Peronnet, On the possible use of active microwave imaging for remote thermal sensing, IEEE Trans. Microwave Theory Tech., 31(9), 777-781, 1983.

Larsen, L. E., and J. H. Jacobi, Microwave scattering imagery of isolated canine kidney, Med. Phys., 6, 394-403, 1979.

Larsen, L. E., and J. H. Jacobi, Microwave time-delay spectroscopic imagery of isolated canine kidney, Med. Phys., 7, 1-7, 1980.

Milder, D. M., and N. H. Wells, Acoustic holography with crossed linear array, IBM J. Res. Dev., 14, 492-500, 1970.

Mueller, R. K., M. Kaveh, and R. D. Iverson, A new approach to acoustic tomography using diffraction techniques, in Acoustic Holography, vol. 8, edited by A. Metherell, pp. 615-628, Plenum, New York, 1978.

Peronnet, G., C. Pichot, J. C. Bolomey, L. Jofre, A. Izadnegahdar, C. Szeles, Y. Michel, J. L. Guerquin-Kern, and M. Gautherie, A microwave diffraction tomography system for biomedical applications, Proc. Eur. Microwave Conf., 13, 529-533, 1983.

Porter, R. P., and S. J. Devaney, Holography and the inverse source problem, J. Opt. Soc. Am., 72(3), 327-330, 1982.

Slattery, B. R., Use of Mills cross receiving arrays in radar systems, Proc. Inst. Electr. Eng., 113(11), 1712-1722, 1966.

J. Ch. Bolomey and G. Peronnet, Groupe d'Electromagnétisme, Laboratoire des Signaux et Systèmes, CNRS/ESE, F-91190 Gif Sur Yvette, France.

L. Jofre, Escola Tecnica Superior d'Enginyers de Telecomunicacio, Calle Jordi Girona Saldago, Barcelona, Spain. 